\title{
Dermatomicosis en población de Manizales
}

\author{
Buitrago G.E.'
}

\section{Resumen}

Se informa sobre el estudio micológico realizado en una población de 1.175 pacientes adultos remitidos por el servicio médico de la ciudad de Manizales. Fue posible establecer diagnóstico positivo en 779 pacientes $(66,3 \%)$, con $72(6,1 \%)$ casos de pitiriasis versicolor, $1(0,1 \%)$ por Tinea nigra palmaris, $291(24,8 \%)$ micosiscutáneaspordermatofitos y $184(15,7 \%)$ casos de candidiasis. La distribución de frecuencia agrupó a 15 Microsporum, 101 Epidermophyton y 257 Trichophyton. Fueron $357(95,7 \%)$ antropofílicos, $10(2,7 \%)$ geofílicos y $6(1,6 \%)$ zoofílicos. Las especies aisladas fueron: M. canis, M. gypseum, E. floccosum, T. rubrum, T. mentagrophytes, T. tonsurans, T. verrucosum, Cladosporium werneckii, Malassezia furtur y otros.

En el estudio de las onicopatías se encontraron: $82(7,0 \%)$ casos por dermatofitos 103 $(8,8 \%)$ casos por Candida albicans; en $46(3,9 \%)$ casos se aislaron hongos no dermatofitos incriminados como agentes patógenos oportunistas, por algunos autores. No hubo diagnóstico micológico en $10(0,8 \%)$ casos por obtenerse en los cultivos Mycelia sterilla, y fueron negativos $386(32,8 \%)$.

Se indican los procedimientos paraanálisis micológico y se mencionanconsideraciones generales sobre la patología de la dermatofitos.

\section{Summary}

This is a report of the mycological study of 1,175 adult patients treate by Manizales medical cervices.

It was possible to confirm a positive diagnosis in 779 patients $(66,3 \%)$, with $72(6,1 \%)$ occurrences of Pityriasis versicolor, $1(0,1 \%)$ of Tinnea nigra palmaris, $291(24,8 \%)$ of dermato-phyticcutaneous mycosisand $184(15,7 \%)$ casesofcandidiasis. The frequency distribution includes 15 Microsporum, 101 Epidermophyton and 257 Tricophyton. The epidemiologic classification includes the following groups of fungi: antropophylic 357 $(95,7 \%)$, geophytlic $10(2,7 \%)$ and zoophylic $6(1,6 \%)$.

The most commonly found species were: M. canis, M. gypseum, E floccosum, T. rubrum, T. mentagrophytes, T. tonsurans, T. verrucosum, Cladosporium werneckkii, and Malassezia furfur.

This report describes the fungi isolated in dystrophic nails as follows: dermatophytes 82 $(7,0 \%)$ and Candida albicans $103(8,8 \%)$. In $46(3,9 \%)$ patients nordermatophyteswere cultured and incriminated as opportunistic pathogens according to several authors. In $10(0,8 \%)$ samples, no etiological diagnosis was obtained because of the growth of Mycelia sterila. No growth ocurred in $386(32,8 \%)$ cultures.

This article presents an overview of the laboratory procedures and some general considerations concerning the pathology of dermatophytes. 
El estudio de las micosis es un tema de obligada revisión en nuestro medio por constituir una causa de consulta médica, por estar localizados en zona tropical, por la migración constante de sus habitantes y por el contacto repetido de las gentes con el medio ambiente.

De acuerdo con investigaciones adelantadas en muchas regiones del mundo, es muy variable la distribución geográfica y la prevalencia (1) de las distintas especies de hongos potencial o claramente patógenos para el hombre.

Con este trabajo se pretende: a) informar sobre los resultados obtenidos con el examen micológico de muestras tomadas en la consulta rutinaria de pacientes adultos, remitidos por el servicio de especialistas y médicos generales de la ciudad; b) contribuir al reconocimiento y cuantificación de la patología micológica regional.

\section{Materiales y métodos}

La población estudiada corresponde en su gran mayoría a clase media y media baja de empleados y obreros de la ciudad de Manizales; el personal examinado procedente de otros municipios del departamento de Caldas, no sobrepasa el $10 \%$.

Las afecciones estudiadas en los pacientes se pueden catalogar en los siguientes grupos: dermatofitosis $(2,3)$, infecciones cutáneas que afectan cabello, epidermis y uñas, producidas por hongos queratinofílicos denominados dermatofitos; dermatomicosis, producidas por levaduras y otros hongos habitantes del suelo; onicomicosis, producidas por levaduras, dermatofitos y otras especies de hongos.

\section{Muestras}

Se recolectaron las siguientes muestras: a) raspado del borde activo de las áreas cutáneas enfermas; b) cabellos enfermos, arrancados de los bordes de las zonas alopécicas; c) raspado de las zonas ungueales enfermas, con recolección preferencial del detritus caseoso acumulado en el hiponiquio o borde distal del lecho ungueal; d) muestras de material purulento tomadas con asa (4), después de presionar el borde proximal ungueal, matriz de la uña, en caso de perionixis, para hacer frotis en portaobjetos con fines de coloración o examen en fresco.

En general en nuestro laboratorio se aplican las siguientes normas: a) suspender toda mediación una semana antes del muestreo; b) limpiar el área afectada con alcohol de $70^{\circ}$, o con agua destilada estéril; c) raspar la muestra directamente sobre la superficie del medio de cultivo; d) tomar muestra para examen directo.

Las muestras se observan con objetivo seco, montadas entre lámina y laminilla con $\mathrm{KOH}$ al $10 \%$, con $\mathrm{KaOH}$ más tinta azul negra Parker 51 a partes iguales, o con algodón azul lactofenol (Cotton blue).

\section{Medios de cultivo}

Los medios de cultivo empleados de rutina para sembrar las muestras comprenden: a) Sabouraud dextrosa agar; b) suero o plasma humano estéril, o albúmina de huevo para la prueba del tubo germinal; c) Sabouraud dextrosa agar adicionado con cicloheximida y cloranfenicol $(0,5$ y 0,05 $\mathrm{g} / \mathrm{L}$ respectivamente) o DTM, (desarrollado por Taplin) $(5,6)$ paraaislamiento de hongos miceliados; d) Sabouraud más suplementos vitamínicos según la especie sospechada, para lograr su reproducción, en caso de hongos zoofílicos especiales.

La identificación de los dermatofitos y otros hongos, fue practicada de acuerdo con las técnicas de la micología médica (7-11).

\section{Resultados}

No se tuvo en cuenta la clasificación por sexo sino que se fueron incorporando los pacientes de acuerdo con la consulta, que al momento del corte final llegó a 1.175 casos así: hombres 561 $(47,7 \%)$ con una positividad de $397(70,8 \%)$ y mujeres $614(52,3 \%)$ con una positividad de $382(62,2 \%)$. Los resultados se presentan en la tabla 1.

Como resultado del estudio adelantado en 1.175 pacientes se han identificado en nuestra región las siguientes especies: $M$. canis, $M$. gypseum, E. floccosum, T. rubrum, T. mentagrophytes, $T$. tonsurans y $T$. verrucosum, todas ellas especies cosmopolitas según la clasificación ecológica de Rippon; $C$. werneckii, Malassezia furfur y otras. 
Tabla 1. Análisis micológico, según sexo, practicado a pacientes de Manizales, 1976-1992.

\begin{tabular}{lcccccc}
\hline Sexo & Positivos & $\%$ & Negativos & $\%$ & Total & $\%$ \\
\hline Hombres & 397 & 70,8 & 164 & 29,2 & 561 & 47,7 \\
Mujeres & 382 & 62,2 & 232 & 37,8 & 614 & 52,3 \\
\hline Total & 779 & 66,3 & 396 & 33,7 & 1.175 & 100,0 \\
\hline
\end{tabular}

Entre las micosis superficiales se encontraron 72 $(6,1 \%)$ casos de pitiriasis versicolor y $1(0,1 \%)$ de Tinea nigra palmaris.

De las micosis cutáneas se diagnosticaron 291 $(24,8 \%)$ casos de dermatofitosis y $184(15,7 \%)$ de candidiasis. El grupo de las onicomicosis dió un total de $231(19,7 \%)$ casos detallados etiológicamente así: por dermatofitos $82(7,0 \%)$, por Candida albicans 103 (8,8\%), en grupo aparte se tomaron en cuenta $46(3,9 \%)$ aislamientos (Aspergilus 8, Penicillium 34, Cephalosporium 2, Sporothrix schenckii 1 y Hemispora1) de hongos miceliados que han sido considerados por diferentes autores como de significado etiológico, y $10(0,8 \%)$ aislamientos clasificados como Mycelia sterila. Se identificaron además otros mohos y levaduras que por ser considerados contaminantes frecuentes, no se mencionan aquí.

El total de casos positivos fue de 779 (66,3\%), cuyo resumen se incluye en la tabla 2 .

En dermatofitosis, el hallazgo más frecuente correspondió a Trichophyton mentagrophytes 184 $(49,3 \%)$, seguido por E. floccosum $10(27,1 \%)$ y T. rubrum $70(18,8 \%)$ del total de 373 casos confirmados. Debe tenerse en cuenta que la población atendida corresponde a adultos. Las especies de dermatofitos identificadas se relacionan en la tabla 3.

Entre los microorganismos no dermatofitos aislados de los 406 casos restantes positivos, se encontraron en orden de frecuencia: Candida albicans $287(24,4 \%)$, Malassezia furfur $72(6,1 \%)$ y otros hongos $46(3,9 \%)$. Los aislamientos no identificados que aparecen como Mycelia sterila
Tabla 2. Afecciones dérmicas según localización, investigadas en pacientes de Manizales, 1976-1992.

\begin{tabular}{llrr}
\hline $\begin{array}{l}\text { Clasificación } \\
\text { clínica }\end{array}$ & \multicolumn{1}{c}{ Entidad } & $\begin{array}{c}\text { Casos } \\
\text { estudiados }\end{array}$ & $\%$ \\
\hline Superficiales & $\begin{array}{l}\text { Pitiriasis versicolor } \\
\text { Tinea nigra palmaris }\end{array}$ & 72 & 6,1 \\
Cutáneas & Dermatofitos & 291 & 0,1 \\
& Candidiasis & 184 & 15,7 \\
Onicomicosis & Dermatofitos & 82 & 7,0 \\
& Hongos no dermatofitos & 46 & 3,9 \\
& Candida albicans & 103 & 8,8 \\
\multirow{2}{*}{ Sin diagnóstico } & Mycelia sterilla & 10 & 0,8 \\
& Micológico negativo & 386 & 32,8 \\
\hline Total & & 1.175 & 100,0 \\
\hline
\end{tabular}

fueron $10(0,8 \%)$. Los resultados se relacionan en la tabla 7.

De acuerdo con la clasificación clínica de los 373 dermatofitos aislados, se encuentra una incidencia de $287(76,9 \%)$ aislamientos de piel, $4(1,1 \%)$ de cabello y $82(22,0 \%)$ de uñas; los detalles se relacionana en la tabla 3 .

Al clasificar, desde el punto de vista epidemiológico, los dermatofitos identificados, se encuentra la siguiente distribución: antropofílicos 357 (95,7\%), Geofílicos $10(2,7 \%)$ y zoofílicos $6(1,6 \%)$. Los detalles por especie se resumen en la tabla 4 .

Delos 1.175 pacientes estudiados, seaisló Candida albicans en 287 casos; sus condiciones patológicas y culturales permitieron clasificarlos como candidiasis; los intertrigos sumaron 143 casos $(49,8 \%)$; oniquia y paroniquia 103 casos $(35,9 \%)$; de tipo cutáneo psoriasiforme $31(10,9 \%)$ casos, queilitis angular 4 casos $(1,4 \%)$, balanitis 5 casos $(1,7 \%)$ y otitis externa $1(0,3 \%)$ caso. Se resumen estos datos en la tabla 5 .

En los análisis de laboratorio realizados a 316 pacientes con onicopatías, el mayor número de aislamientos específicos correspondió a Candida albicans (103) con un 32,6\% y una frecuencia mucho mayor en manos $65(63,1 \%)$ que en uñas de los pies, $38(36,9 \%)$. 
Tabla 3. Dermatofitos aislados según estructuras afectadas en pacientes de Manizales, 1976-1992.

\begin{tabular}{|c|c|c|c|c|c|c|c|c|c|}
\hline & \multicolumn{2}{|c|}{ Microsporum } & \multirow{2}{*}{$\begin{array}{c}\text { Epidermophyton } \\
\text { floccosum }\end{array}$} & \multicolumn{3}{|c|}{ Trichophyton } & & \multirow[t]{2}{*}{ Total } & \multirow[t]{2}{*}{$\%$} \\
\hline & canis & gypseum & & metagrophytes & rubrum & tonsura & ucosum & & \\
\hline Piel & 3 & 10 & 92 & 125 & 56 & 0 & 1 & 287 & 76,9 \\
\hline Cabello & 2 & 0 & 0 & 0 & 0 & 2 & 0 & 4 & 1,1 \\
\hline Uñas & 0 & 0 & 9 & 59 & 14 & 0 & 0 & 82 & 22,0 \\
\hline Total & 5 & 10 & 101 & 184 & 70 & 2 & 1 & 373 & 100,0 \\
\hline
\end{tabular}

Tabla 4. Clasificación epidemiológica de los dermatofitos aislados en pacientes de Manizales, 1976-1992.

\begin{tabular}{|c|c|c|c|c|c|}
\hline Grupo & Especie & Cultivos & $\%$ & Total grupo & $\%$ \\
\hline \multirow[t]{4}{*}{ Antropofílicos } & E. floccosum & 101 & 27,1 & & \\
\hline & T. mentagrophytes & 184 & 49,3 & 357 & 95,7 \\
\hline & T. rubrum & 70 & 18,8 & & \\
\hline & $T$ tonsurans & 2 & 0,5 & & \\
\hline \multirow[t]{2}{*}{ Zoofílicos } & M. canis & 5 & 1,3 & & \\
\hline & T. verrucosum & 1 & 0,3 & 6 & 1,6 \\
\hline Geofílicos & M. gypseum & 10 & 2,7 & 10 & 2,7 \\
\hline Total & & 373 & 100,0 & 373 & 100,0 \\
\hline
\end{tabular}

Tabla 5. Muestras positivas para Candida albicans, tomadas a pacientes de Manizales, 1976-1992.

\begin{tabular}{llrr}
\hline Entidad clínica & Región & $\mathbf{n}$ & $\%$ \\
\hline Oniquia y & Pies & 34 & 11,8 \\
paroniquia & Manos & 69 & 24,0 \\
Intertrigo & Interdigital pies & 83 & 29,0 \\
& Mamario & 1 & 0,3 \\
& Axilar & 6 & 2,1 \\
& Crural & 3 & 1,1 \\
Queilitis angularis & Inguinal & 50 & 17,4 \\
Piel & & 4 & 1,4 \\
Balanitis & & 31 & 10,9 \\
Otitis externa & & 5 & 1,7 \\
\hline Total & 287 & 100,0 & 0,3 \\
\hline
\end{tabular}

Entre los dermatofitos aislados (82) con un porcentaje del $26 \%$, la mayor frecuencia correspondió a $T$. mentagrophytes $59(71,9 \%)$ y en mucho menor cantidad T. rubrum $14(17,1 \%)$ y E. floccosum $9(11,0 \%)$ (tabla 7$)$.

Se formó un grupo con hongos miceliados no dermatofitos aislados en el estudio, teniendo en cuenta que diversos autores $(13,14)$ los relacionan como agentes etiológicos de onicomicosis; dicho grupo alcanza a constituir un $14,5 \%$ con la siguiente distribución: Penicillium 34, Aspergillus 8, Cephalosporium 2, Hemispora 1 y Sporothrix schenckii 1.

Por no tener clasificación definida, se agrupan 10 $(0,8 \%)$ aislamientos bajo el título de Mycelia sterila, y como cultivos negativos, un total de 85 $(26,9 \%)$. La distribución de las especies aisladas se resume en las tablas 6 y 7 . 
Tabla 6. Análisis micológico de onicopatías, en pacientes de Manizales, 1976-1992.

\begin{tabular}{lcccccc}
\hline Agente etiológico & Manos & $\%$ & Pies & $\%$ & Total & $\%$ \\
\hline Dermatofitos & 15 & 18,3 & 67 & 81,7 & 82 & 26,0 \\
C. albicans & 65 & 63,1 & 38 & 36,9 & 103 & 32,6 \\
Otros hongos & 14 & 30,4 & 32 & 69,6 & 46 & 14,5 \\
Cultivos negativos & 47 & 55,3 & 38 & 44,7 & 85 & 26,9 \\
\hline Total & 141 & 44,6 & 175 & 55,4 & 316 & 100,0 \\
\hline
\end{tabular}

Tabla 7. Especies aisladas de onicopatías, en pacientes de Manizales, 1972-1992.

\begin{tabular}{|c|c|c|c|c|c|c|}
\hline & Manos & $\%$ & Pies & $\%$ & Total & $\%$ \\
\hline \multicolumn{7}{|l|}{ DERMATOFITOS } \\
\hline T. mentagrophytes & 12 & 20,3 & 47 & 79,7 & 59 & 71,9 \\
\hline T. rubrum & 3 & 21,4 & 11 & 78,6 & 14 & 17,1 \\
\hline E. floccosum & 0 & 0 & 9 & 100,0 & 9 & 11,0 \\
\hline Total & 15 & 18,3 & 67 & 81,7 & 82 & 100,0 \\
\hline \multicolumn{7}{|l|}{ LEVADURAS } \\
\hline C. albicans & 65 & 63,1 & 38 & 36,9 & 103 & 100,0 \\
\hline \multicolumn{7}{|l|}{ OTROS HONGOS } \\
\hline Aspergillus sp. & 1 & 12,5 & 7 & 87,5 & 8 & 17,4 \\
\hline Penicillium sp. & 11 & 32,4 & 23 & 67,6 & 34 & 73,9 \\
\hline Hemispora sp. & 1 & 100,0 & 0 & 0 & 1 & 2,2 \\
\hline Cephalosporium sp. & 1 & 50,0 & 1 & 50,0 & 2 & 4,3 \\
\hline Sporothrix schenckii & 0 & 0 & 1 & 100,0 & 1 & 2,2 \\
\hline Total & 14 & 30,4 & 32 & 69,6 & 46 & 100,0 \\
\hline Cultivos negativos & 47 & 55,3 & 38 & 44,7 & 85 & 100,0 \\
\hline
\end{tabular}

\section{Discusión}

De las micosis superficiales llama la atención la pitiriasis versicolor con 72 casos nuestros, hecho fácilmente explicable porque es una enfermedad común en climas tropicales; en Caldas es frecuente en zonas limítrofes de los ríos Magdalena y Cauca.

Marcon y Powel (15) informan sobre el carácter patógeno oportunista de la Malassezia furfur en pacientes con cateterismo vascular profundo e hiperalimentación parenteral. El uso de emulsiones grasas intravenosas parece haber alterado el microambiente del catéter y permitido la localización e infección subsecuente. Luego debe mantenerse un control clínico de pacientes que concomitantemente presente pitiriasis versicolor, con un alto índice de sospecha de sepsis del carácter por Malassezia furfur en pacientes hiperalimentados parenteralmente.

Powell y col. (16) estudiaron la prevalencia de Malassezia furfur en la colonización cutánea de los niños hospitalizados en unidades de cuidado intensivo neonatal y de cirugía cardiaca. De 361 niños estudiados en un año, $133(36,8 \%)$ tuvieron, por lo menos, un resultado positivo. 
La presentación de un caso de Tinea nigra palmaris indica posiblemente su baja frecuencia en Caldas, al contrario de la costa atlántica, donde ha sido informada como hallazgo frecuente (17); en otras regiones colombianas se ha encontrado la localización plantar de la afección (18).

De los 1.175 casos nuestros, 316 (26,9\%) fueron remitidos por presentar onicopatías; los $859(73,1 \%)$ casos restantes que constituyeron los tres cuartos del volumen total, fueron remitidos con sospecha de micosis cutáneas.

Los dermatofitos deben tomarse en cuenta en infecciones hospitalarias; Mossovitch y col. (19) informan que durante las labores de alimentación artificial, un niño que sufría una lesión por $M$. canis en la región occipital, transmitió el hongo al antebrazo izquierdo de 7 enfermeras. Neves y Xavier (20) fueron capaces de aislar E. floccosum y $T$. rubrum no solamente de los vestidos y las sábanas de los pacientes en una sala hospitalaria, sino también de su medio ambiente (orinales, servicios sanitarios y cómodas).

Se diagnosticaron $143(49,8 \%)$ casos de intertrigo siendo los más frecuentes interdigital de pies (83) e inguinal (50). De los casos de oniquia y paroniquia, 69 corresponden a las manos y $34 \mathrm{a}$ los pies; el número mayor en manos se explica por razones de ocupación (lavanderas, empleadas de bares, restaurantes, empacadores de alimentos, etc.) y empleo constante de agua, jabones, detergentes y la irritación debida a jugos de frutas y vegetales. (tabla 5).

Al manejar este tipo de patología, siempre debemos pensar en al existencia de las enfermedades de base, con miras a un buen diagnóstico y una mejor orientación del tratamiento. En la tabla 8 se resumen los factores que favorecen la candidiasis.

El mayor número de aislamientos correspondió a Candida albicans con 103 (32,6\%), seguido por $82(86,0 \%)$ casos de dermatofitos con gran predominio de $T$. mentagrophytes y de mayor localización en uñas de los pies.

En un estudio realizado en Medellín por Restrepo y col.(21) en 3.818 pacientes con lesiones de piel $y$ anexos, $639(16,7 \%)$ correspondieron a candidiasis; encontraron 224 pacientes con lesiones en uñas y 60 con lesiones periungueales; entre los 224 casos encontraron $160(71,4 \%)$ con lesiones de tipo onicolisis (desprendimiento de la uña del lecho ungueal y acumulación de detritus).

El panorama de hongos saprofitos oportunistas $(22,23)$ se ha venido modificando en los últimos años, con la presentación de casos producidos por agentes nuevos debido a variaciones fisiológicas de índole medicamentoso o inmunológico. Di Silverio (24) y Stenderup (25) informaron sobre alternariosis cutáneas en pacientes inmunosuprimidos a consecuencia de otras patologías.

Tabla 8. Factores que favorecen la candidiasis.

1. Intrínsecos

2. Extrínsecos
Fisiológicos

Patológicos

Medicamentos

Quirúrgicos

Físicos

Otros factores
Edad (recién nacido, ancianos), embarazo

Diabetes, hipotiroidismo, obesidad, enfermedades consuntivas, malnutrición, trastornos dermatológicos, carcinomas, leucemia, alteraciones inmunitarias, deficiencias genéticas.

Inmunosupresores, corticoides, citotóxicos, anticonceptivos orales, antibacterianos de amplio espectro.

Cateterismo, diálisis peritoneal, trasplantes, traqueostomías.

Rayos $\mathrm{X}$, quemaduras, traumatismos, humedad, exposición ocupacional.

Maceración, prótesis defectuosas, drogadicción, infecciones. 
Gentles y Evans (26) informan sobre infección de pies de uñas por Hendersonula toruloidea; Di Salvo (27) confirma un caso de onicomicosis por Fusarium oxysporum, hongo no dermatofítico; Campbell (28) estudia el Scytalidum hyalinum; Restrepo y Uribe (29) aislan hongos de los géneros Geotrichum y Trichosporum de lesiones dérmicas, con fundamentada conclusión sobre su papel etiológico; y así, van haciendo incursión especies que antes no se consideraban con patogenicidad suficiente para el huésped humano; estos hallazgos deben mantenernos alerta para cuando aislemos agentes nuevos, en ausencia de los tradicionalmente patógenos. Por otra parte, los estudios del medio ambiente siguen enriqueciendo la lista con especies qeratinofílicas nuevas, como sucede por ejemplo con las citas de Borelli (30) sobre Microsporum racemosum y Trichophyton phaseoliforme, de Moraes (31) sobre Microsporum amazonicum, o de Stiller y col. (32) sobre onicomicosis causada por Chaetomium globosum.

En el estudio se informa sobre el aislamiento de 45 hongos miceliados, que al revisar la literatura disponible, figuran incriminados por diferentes autores como agente etiológicos de importancia. El aislamiento de estos hongos debe tener una doble evaluación: en el laboratorio, observar que en el cultivo de la muestra, aparezca buen número de colonias del hongo específico, compatible con los hallazgos al examen microscópico directo; que el mismo hongo sea aislado de tres muestras tomadas en días diferentes, ya que los hongos miceliados no se establecen como flora normal permanente en las superficies del cuerpo humano; que la morfología vegetativa del hongo se demuestre en exámenes microscópicos directos como invasor de los tejidos; y que no se aisle un microorganismo semejante como undermatofito, a pesar de muestreos repetidos. En la tabla 7 se enumeran los agentes aislados de las onicopatías.

\section{Referencias}

1. Allejo L. Natural history of the dermatophytes and related fungi. Mycopath Mycol Appl 1974; 53:93.

2. Beare JM, Gentler JC, et al. Textbook of dermatology. Chapter 25. Book A, et al. (eds) Philadelphia, 1972.
3. Conant NF, Smith DT, Baker RD, et al. Chapter LXXV. Manual of Clinical Mycology. 3rd ed.Philadelphia: W.B. Saunders, 1971: 595.

4. Huppert M, Harper G, Sung SH, et al. Rapid methods of identification of yeasts. J Clin Microbiol 1975; 2:21.

5. Rebell G, Taplin D. Dermatophytes: their recognition and identification. 2nd ed. Miami: Coral Gables, Fla. U. Press, 1970.

6. Taplin D, Zaias N, Rebell G, et al. Isolation and recognition of dermatophytes on a new media. Arch Dermatol 1969; 99:203

7. Ajello L, Georg IK. In vitro hair cultures for differentiating between atypical isolates of $T$. mentagrophytes and $T$. rubrum. Mycopath 1957; 8:3.

8. Dolan CT. A practical approach to identification of yeastlike organisms. Am J Clin Pathol 1971; 55:580.

9. Georg LK, Camp LE. Routine nutritional tests for the identification of dermatophytes. J Bacteriol 1957; 74:477.

10. Hazen EL, Reed FC. Laboratory identification of pathogenic fungi simplified. Springfield: Charles C. Thomas Publisher. 1955:I11

11. Moore GS, Jaciow DM. Yeast identification. The opportunistic fungi. Chapter 3. 34. In: Mycology for the clinical laboratory. Reston Publishing Co, Inc 1979.

12. Rippon JW. Dermatofitosis y dermatomicosis. En: Tratado de Micología Médica. 3a. ed. Interamericana. New York: McGraw Hill, 1988:195.

13. Conti-Díaz IA. Estudio micológico de 85 casos de onicopatías. An Fac Medic Montevideo 1964; 49:5.

14. Zaias N. Onychomycosis. Arch Dermatol 1972; 105: 263.

15. Marcon MJ, Powell DA. Epidemiology, diagnosis and management of Malassezia furfur systemic infection. Diagn Microbiol Infect Dis 1987; 7:161.

16. Powell DA, Hayes J, Durrell DE, et al. Malassezia furfur skin colonization of infants hospitalized in intensive care units. J Pediatr 1987; 111:217.

17. Gómez-Sierra H, Cárdenas JV, Rendón-Pizano I. Tinea nigra. Mycopath Mycol Appl 1968; 34:11.

18. Rueda-Plata LA, Rodríguez-Pelayo R. Tinea nigra plantaris. Primer caso observado en Colombia. Presentado en el IV Congreso Colombiano de Microbiología. Manizales. 1968.

19. Mossovitch M, Mossovitcht B, Alkan M. Nosocomial dermatophytosis cause by Microsporum canis in a newborn department. Infect Control 1986; 7:593.

20. Neves $\mathbf{H}$, Xavier NC. The transmission of Tinea cruris. Brit J Derm 1964; 76:429.

21. Restrepo A, Moncada LH, Quintero M, et al. Candidiasis. La multiplicidad de sus manifestaciones clínicas. Tribuna Médica $1973 ; 47: 15$.

22. Philpot CHM. Some aspects of the epidemiology of tinea. Mycopathol 1977; 62:3. 
23. Rogers AL. Hongos saprofitos oportunistas y contaminantes. En: Lennette EH, Balows A, Hausler WJ, et al. eds. Manual de microbiología clínica, 3a. ed. Editorial Médica Panamericana S.A., 1982:785.

24. Di Silverio A, Sacchi S. Cutaneous alternairosis: a rare chromohyphomycosis. Report of a case. Mycopatho 1986; 95:159.

25. Stenderup J, Bruhn M, Gadeberg G, et al. Cutaneous alternariosis. Case report. Act Pathol Microbiol Inmunol Scand 1987; 95:79.

26. Gentles JC, Evans EGV. Infection of the feet and nails with Hendersonula toruloidea. Sabouraudia 1970; 8: 72.

27. Di Salvo A, Fickling AM. A Case of nondermatophytic toe onychomycosis caused by Fusarium oxysporum. Arch Dermatol 1980; 116:669.
28. Campbell CK, Mulder JL. Skin and nail infection by Scytalidium hyalinum sp. nov. Sabouradia 1977; 15: 161.

29. Restrepo A, de Uribe $L$. Isolation of fungi belonging to the genera Geotrichum and Trichosporum from human dermal lesions. Mycopathol 1976; 59:3.

30. Borelli D, Feo M. Nota previa sobre un nuevo queratinomiceto: Trichophyton phaseoliforme Acta Med Venezolana. 1966; 13:176.

31. Morales M, Borelli D, Feo M. Microsporum amazonicum nova species. Medicina Cutánea 1987; 2:281.

32. Stiller MJ, Rosenthal S, Summerbell RC, et al. Onychomycosis of the toenails caused by Chaetomium globosum. J Am Acad Dermatol 1992; 26:775. 